\title{
FTIR spectroscopic imaging and mapping with correcting lenses for studies of biological cells and tissues
}

\author{
James A. Kimber ${ }^{a}$, Liberty Foreman ${ }^{b}$, Peter Rich ${ }^{b}$ and Sergei G. Kazarian ${ }^{* a}$ \\ a. Department of Chemical Engineering, Imperial College London, London, SW7 2AZ \\ b. The Glynn Laboratory of Bioenergetics, Institute of Structural and Molecular Biology, University College London, London, WC1E 6BT
}

\begin{abstract}
Histopathology of tissue samples is used to determine the progression of cancer usually by staining and visual analysis. It is recognised that disease progression from healthy tissue to cancerous is accompanied by spectral signature changes in the infrared range. In this work, FTIR spectroscopic imaging in transmission using a focal plane array ( $96 \times 96$ pixels) has been applied to the characterisation of Barrett's esophageal adenocarcinoma. Spectral images were acquired in transmission mode. To correct optical aberrations, infrared transparent lenses were used of the same material as the slide on which biopsies were fixed. The lenses acted as an immersion objective, reducing scattering and improving spatial resolution.
\end{abstract}

A novel mapping approach is presented where spectral images obtained with added lens are stitched together such that the dataset contained a representative section of the esophageal tissue. Images were also acquired in transmission mode using high-magnification optics for enhanced spatial resolution, and were also acquired using a germanium micro-ATR objective for comparison. The reduction of scattering was assessed using k-means clustering. The same tissue section map, which contained a region of high grade dysplasia was analysed using hierarchical clustering analysis. A reduction of the trough at $1077 \mathrm{~cm}^{-1}$ in the second derivative spectra was identified as an indicator of high grade dysplasia.

In addition, the spatial resolution obtained with the added lens using high-magnification optics was assessed by measurements of a sharp interface of polymer laminate, which was also compared with the spatial resolution achieved in micro ATR-FTIR imaging. The resolution was determined from the change in absorbance of spectral bands from one side of the polymer interface to the other. In transmission mode using the lens, it was determined to be $12 \mu \mathrm{m}$ and using micro-ATR, the resolution was $3 \mu \mathrm{m}$ for the band at ca. $6 \mu \mathrm{m}$ The spatial resolution was also assessed with and without the added lens, in normal and high-magnification mode using a USAF target.

Spectroscopic images of cells in transmission using two lenses are also presented, which are necessary for correcting chromatic aberration and refraction in both the condenser and objective. The use of lenses is shown to be necessary in obtaining high-quality spectroscopic images of cells in transmission mode and proves the applicability of the pseudo hemisphere approach for this and other microfluidic systems. 


\section{Introduction}

Fourier Transform Infrared (FTIR) mid-infrared micro-spectroscopy combined with a focal plane array detector, ${ }^{1-4}$ typically containing $64 \times 64$ pixels, has been used to characterise a variety of materials and systems such as polymers, ${ }^{5-8}$ crude oil deposits $^{9,10}$ and microfluidic devices. ${ }^{11,12}$ The acquisition of spatial and chemical information simultaneously allows for the measurement of heterogeneous samples at a significantly faster rate than would be possible with point mapping. In particular, FTIR spectroscopic imaging is suitable for the analysis of cellular tissue and biological materials ${ }^{13,14}$ due to its intrinsic label-free approach, requiring no modification of the tissue unlike the staining methods commonly used for visible assessment and can reveal information on protein conformation ${ }^{15}$ and other cellular components. ${ }^{16,17}$ This powerful approach, which relies on molecular vibrations, can be used to differentiate disease states of cells ${ }^{18}$ and thus is of great interest with respect to new diagnostic techniques as it can remove some degree of subjectivity that is present with visual histopathology. ${ }^{19-21}$

Measurement of tissue samples using FTIR spectroscopic imaging is typically done in transmission mode $^{22,23}$ or attenuated total reflection (ATR) mode. ${ }^{14,16}$ Both sampling approaches have their advantages and disadvantages and the choice of one approach over the other depends on the sample being analysed. ${ }^{3,16,24}$ ATR mode uses a high-refractive index prism such that infrared light undergoes total internal reflection. It probes samples using the evanescent wave, which penetrates into the sample between $1 \mu \mathrm{m}$ and $10 \mu \mathrm{m}$ depending on the wavelength of light, angle of incidence and refractive indices of the sample and prism. As such, it is independent of overall sample thickness and offers higher spatial resolution due to the use of high-refractive index materials in the prism (e.g. germanium) which lowers the diffraction limit because of increased numerical aperture., 14 With respect to cellular imaging, isolated cells can be studied in detail, with a projected pixel size of $1 \mu \mathrm{m}$ and field of view of approximately $64 \times 64 \mu \mathrm{m}^{2}$. However, ATR mode is a contact approach which has the possibility of deforming or damaging the sample such as a tissue, and introducing cross-contamination between measured regions, causing mapping to be particularly challenging.

Transmission mode allows for contact-free mapping with the use of an XY stage, and a wider field of view of $170 \times 170 \mu \mathrm{m}^{2}$ with a projected pixel size of $2.67 \mu \mathrm{m} .{ }^{25}$ Advances in microscope design such as with the Agilent Cary 620 system use 'high-magnification' (HM) optics, producing a projected pixel size of $1.1 \mu \mathrm{m}$ - comparable to $\mathrm{ATR}^{26}$. However, the real spatial resolution of the system, R, i.e. the distinct resolution between two points (airy discs), is determined by the Rayleigh criterion (Eq. 1), which itself is a function of the numerical aperture of the condenser $\left(N A_{c}\right)$ and objective $\left(N A_{\circ}\right){ }^{27}$ Numerical aperture is defined as $n \sin \theta, \lambda$ is the wavelength of light and $\mathrm{R}$ is the distance between two points.

$R=\frac{1.22 \lambda}{N A_{O}+N A_{C}}$

Often the numerical aperture of the objective and condenser are the same so that at $3000 \mathrm{~cm}^{-1}$, the maximum spatial resolution for a microscope with a $0.62 \mathrm{NA}$ objective and condenser is $3.3 \mu \mathrm{m}$. As the FPA is spatially discrete, it is necessary to oversample the imaged area to avoid aliasing effects and obtain true diffraction-limited images; hence the use of HM optics can reveal details at this limit. The Agilent high-magnification system produces projected pixel size of $1.1 \mu \mathrm{m}$ with $15 x$ objective by introducing additional $5 \mathrm{x}$ magnification optics that also includes $2.06 \mathrm{x}$ de-magnification unit resulting on overall $36 \mathrm{x}$ magnification, so dividing the physical size of pixel in FPA detector by 36 
produces the value of $1.1 \mu \mathrm{m}$. This projected pixel size has been cited by others as "spatial resolution" which is imprecise and thus needs to be stated clearly that this value is significant overestimation of the achieved spatial resolution. Bhargava and co-workers ${ }^{28}$ produced a recent report for practitioners of FTIR imaging about the effects of optical components on measured spectra as guidance for optimisation of FTIR imaging systems. They highlighted that this is particularly important for biomedical analysis since scattering effects when measuring tissue samples affect quality of obtained spectra. ${ }^{29-31}$ Our methodology presented here is one of the practical approaches for improving FTIR spectra of tissues and cells by using additional optical components in imaging systems.

Transmission mode in FTIR spectroscopic imaging relies on infrared light passing through the whole sample and can have a higher signal to noise compared with ATR mode. Its limitation is the requirement for thin samples to avoid saturation of spectral bands and also because thickness of the samples should typically be less than the average size of domains as otherwise it could produce a spurious image where domains overlap. Another disadvantage of transmission mode is the potential for optical effects that degrade image and spectral quality. One such effect is scattering which produces spectral anomalies and degrade spatial resolution. For biological samples, scattering is predominantly Mie and resonant Mie scattering which is due to cells and their organelles being of similar dimensions to the wavelength of mid-infrared light (between 3 to $12 \mu \mathrm{m}$ ). ${ }^{30,32,33}$ These distortions manifest themselves in the spectrum and interfere with subtle band shifts often purported to be due to changes in protein morphology. This is particularly problematic around the Amide I band where a particularly strong dispersion artefact exists. Existing methods to correct spectra such as extended multiplicative scatter correction (EMSC) algorithms, and more recently, iterative resonant Mie scattering EMSC (RMieS-EMSC) have been used to correct scattering and the aforementioned dispersion artefact. ${ }^{30,34}$

Spectroscopic imaging in transmission through spectroscopic windows also introduces chromatic aberration, where different wavelengths are in focus at different distances from the objective. This is due to infrared light entering the window at an angle, causing degrees of refraction as a function of wavelength. This can be corrected using a lens of the same material, placed on top of the window to form a pseudo-hemisphere. ${ }^{25}$ In this way, light enters normal to the lens surface and does not refract at the entry to the window material as the refractive indexes of the lens and window are the same. This removes refraction and chromatic aberration, and acts as an immersion objective which increases the spatial resolution. Chan and Kazarian demonstrated this pseudo-hemisphere approach (Figure 1) with calcium fluoride lenses, where the spatial resolution was increased by a factor of 1.4 for both a polymer interface and a 1951 USAF target. ${ }^{25}$ A potential issue with the use of a correcting lens is the reduction in the field of view. In cases without the lens, high resolution and large field of views can be obtained by mapping the sample, obtaining spectral images which are tiled to form a large area. This is important as mapping large areas automatically can obtain from thousands to millions of spectra for use with multivariate statistical analysis. ${ }^{35}$ In this work, we demonstrate a method by which to map samples with the lens in place, increasing the spatial resolution and providing large area imaging capabilities. It was also reported that scattering is reduced using the lens, ${ }^{25,36}$ the degree of which is also assessed in this work when imaging with added lens combined with mapping approach. We also present the use of the lens with and without HM mode for the first time. 


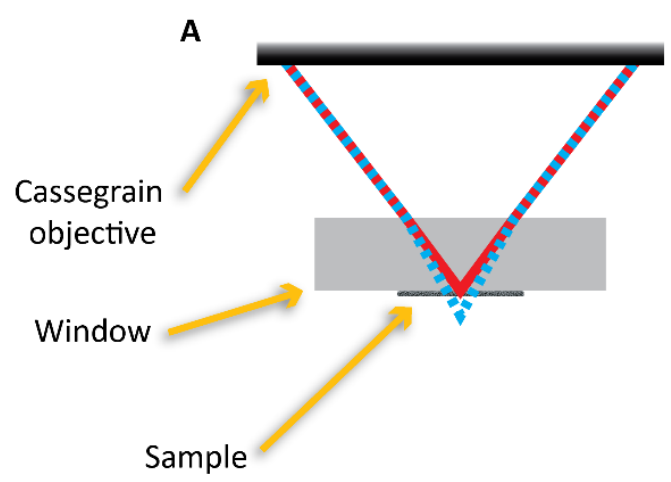

B

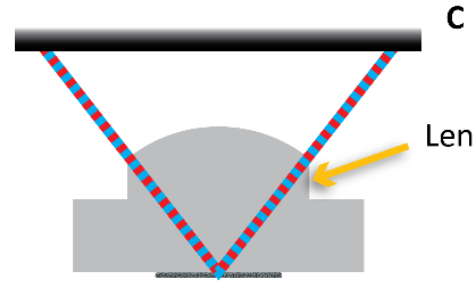

C

Cassegrain condenser

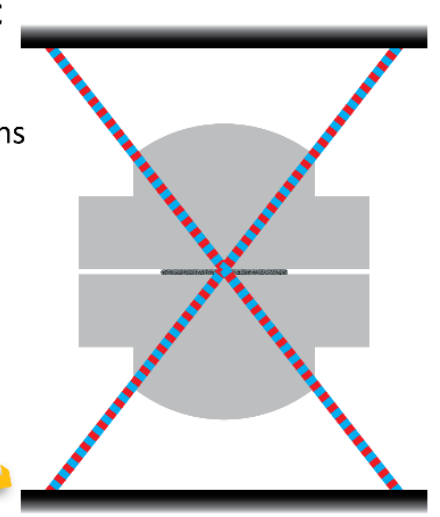

Figure 1 - Schematic of chromatic aberration through an infrared window (A) which is corrected with the use of a lens $(B)$. The lens and window, both made of the same material, form a pseudo-hemisphere, acting as an immersion objective and increasing spatial resolution. For samples between two windows such as a microfluidic device, a bottom lens can be added for the condenser (C).

\section{Methodology}

A tissue sample as also used by Foreman et al was used for these experiments. ${ }^{37}$ In brief, a oesophageal biopsy section of $8 \mu \mathrm{m}$ thickness was mounted on a $2 \mathrm{~mm}$ thick calcium fluoride window. The sample was deparaffinised as per the procedure described by Foreman et al and stored with desiccant before use. For spatial resolution tests, a 1951 USAF resolution .target made from $1.5 \mathrm{~mm}$ float glass and vacuum deposited chromium supplied by Edmund Optics was used. As the spatial resolution using a USAF target is a highly idealised system, a $6 \mu \mathrm{m}$ thick sample containing a sharp interface between two dissimilar polymers was also imaged using the lenses in HM mode. The polymer interface, acting as a representative real sample, creates a test by which spectral contamination from one side of the interface to the other can be assessed, providing a measure of spatial resolution relevant to spectroscopic imaging of tissues and as noted in previous work, is actually 3 times worse than the resolution reported by the USAF target. ${ }^{25}$

Mapping experiments were conducted using a Bruker IRscope II infrared microscope with a $15 \times 0.4$ NA objective and 96x96 FPA detector, connected to a Bruker IFS 66 spectrometer. All other experiments were measured using an Agilent Cary 620 microscope with a 15x 0.62 NA objective with HM optics where stated, connected to an Agilent 670 spectrometer. Tissue samples were measured at $4 \mathrm{~cm}^{-1}$ spectral resolution and with 192 scans. USAF target and polymer interface measurements were measured at $8 \mathrm{~cm}^{-1}$ with 128 scans.

The tissue sample was measured either with the tissue on the top-side of the calcium fluoride window for mapping without the lens i.e. tissue closest to the objective, and inverted for mapping with the lens, i.e. tissue closest to the condenser. The lens as used by Chan and Kazarian ${ }^{25}$ was also applied in this work, which was originally designed for $4 \mathrm{~mm}$ thick calcium fluoride windows. In this case, an additional $2 \mathrm{~mm}$ thick window was placed between the mounted sample window and the lens to place the tissue at the lens focal point. To avoid off-centre distortions such as coma, the field aperture of the IRscope II was used to centre the lens and condenser with the objective. Placing the lens in a custom-designed holder ensured that the lens remained in alignment even if the window 


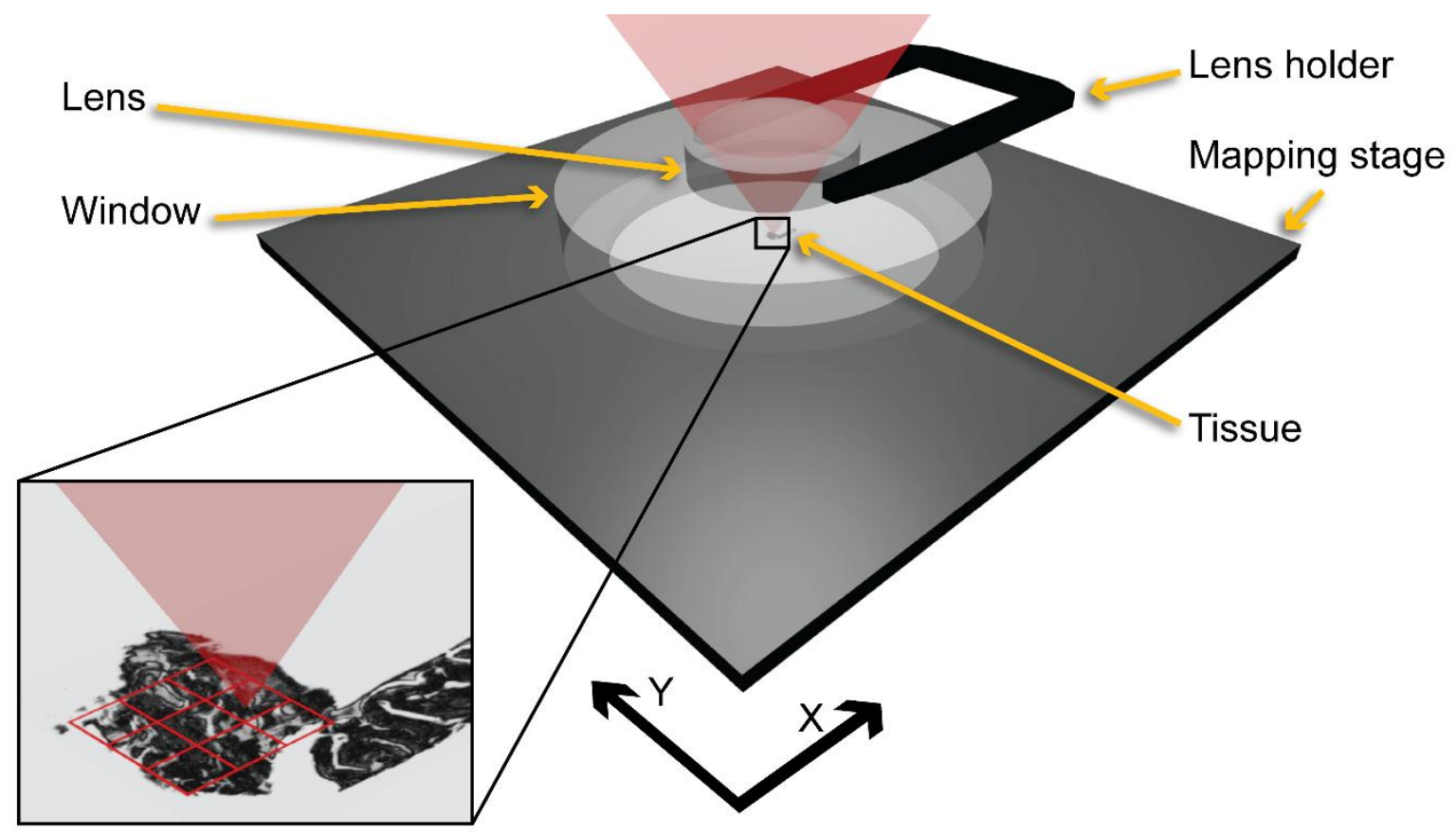

Figure 2 - Schematic showing imaging and mapping approach using the lens. The lens is kept in line with the objective while the window and sample are slid underneath using an XY stage, obtaining multiple tiles which are subsequently combined to form a large spectral image.

with the attached sample was moved by the XY stage. In this way, the window with the sample could slide underneath the lens, enabling mapping (Figure 2). Custom OPUS ${ }^{\circledR}$ macros to control the stage and take measurements were used to obtain separate tiles. A blank region was marked for acquiring a background such that every $\mathrm{N}$ scans, a background tile would also be measured. As the background would change over time, each tile was ratioed against a linear interpolation of the two nearest (in time) background tiles to produce absorbance spectra. Tiles were then stitched together to produce a large array of spatially resolved absorbance spectra. The software used for imaging datasets ratioing and stitching was Matlab ${ }^{\circledR}$.

For imaging with and without HM optics on the Cary 620 microscope, the tissue was imaged in a similar way to before but without the lens holder as mapping was not conducted for these experiments. The USAF target was measured with and without the lens, and in cases with the lens, an additional $4 \mathrm{~mm}$ calcium fluoride window was placed between the lens and the USAF target. For the polymer interface sample, it was sandwiched between two $4 \mathrm{~mm}$ calcium fluoride windows, and lenses were placed above and below the windows as was used in our studies of imaging of live cells in microfluidics. The use of two lenses ensures the numerical aperture of the condenser and objective are equal, further increasing the spatial resolution than is possible with only one lens. The polymer was imaged in HM mode.

In order to assess the degree of scattering from the tissue sample with each imaging set-up, spectra were processed using Cytospec software. Initially, spectra were passed through a quality test based on the integrated absorbance of the band corresponding to Amide II $\left(1485 \mathrm{~cm}^{-1}-1570 \mathrm{~cm}^{-1}\right)$, with pixels falling below an integrated absorbance value of $10 \mathrm{~cm}^{-1}$ being rejected from further analysis. Subsequently, spectra were vector-normalised using the region between $1200 \mathrm{~cm}^{-1}$ and $2000 \mathrm{~cm}^{-1}$ i.e. the fingerprint region. Although FPA detector is sensitive to $900 \mathrm{~cm}^{-1}$, the long path-length of calcium fluoride with the lens $(8 \mathrm{~mm})$ significantly reduced light throughput below $1000 \mathrm{~cm}^{-1}$ and as a result there was increased noise between $1000 \mathrm{~cm}^{-1}$ and $1200 \mathrm{~cm}^{-1}$. Finally, k-means clustering to generate 2 clusters was performed on the spectra using the region between $1200 \mathrm{~cm}^{-1}$ and $2500 \mathrm{~cm}^{-}$ ${ }^{1}$, which would capture not only the fingerprint region but also the dispersion artefact. The spectral 
distance method used was the Pearson's correlation coefficient (D-Values). The k-means clustering would thus classify spectra into clusters with and without the dispersion artefact, and average spectra of the clusters from original measured spectra are presented to demonstrate this along with the cluster maps. The integrated absorbance band corresponding to Amide II $\left(1485 \mathrm{~cm}^{-1}-1570 \mathrm{~cm}^{-}\right.$ $\left.{ }^{1}\right)$ was also plotted as a function of all pixels to generate chemical images of the tissues.

\section{Results and discussion}

\section{Mapping with and without the lens}

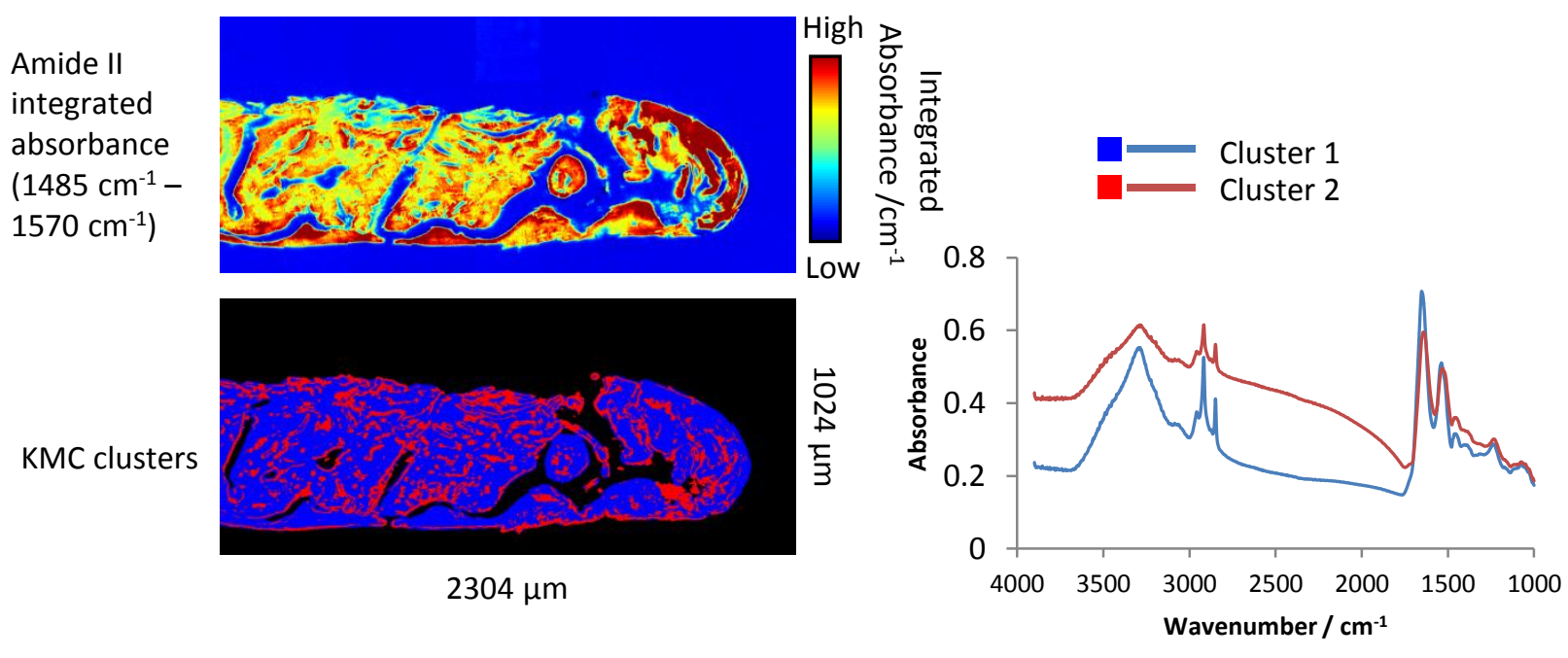

Figure 3 - FTIR image of oesophageal tissue without the lens obtained by mapping from multiple stitched images Clusters from KMC are shown with average spectra from each cluster. Cluster 2 (red) indicates strong scattering at the edges of the tissue.
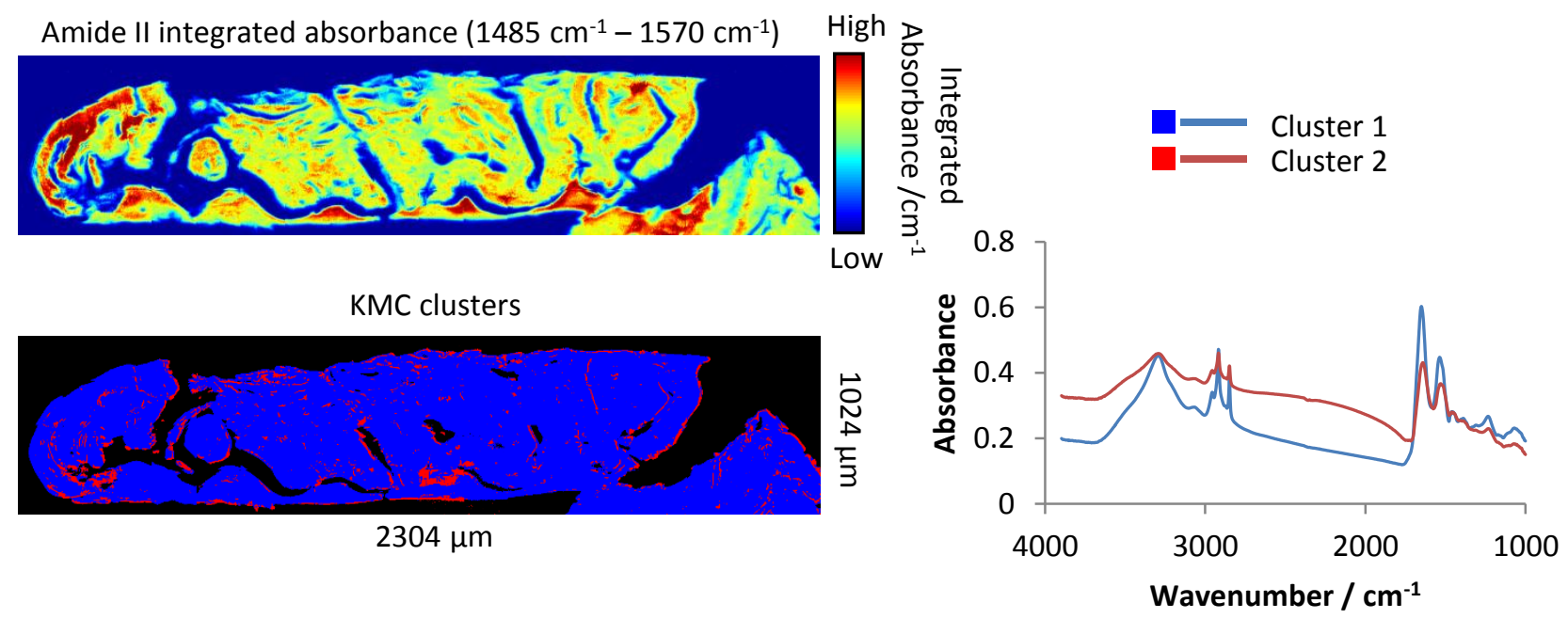

Figure 4 - FTIR image of esophageal tissue with the lens obtained by mapping from multiple stitched images. Clusters from KMC are shown with average spectra from each cluster. Cluster 2 (red) indicates scattering at the edges of the tissue and the fraction of spectra classified into cluster 2 is reduced using the lens.

Figure 3 shows the results of mapping without the lens. K-means clustering was used to sort regions into those without the dispersion artefact and those with, as shown in the average spectra from each cluster (cluster 1 and 2 respectively). Although a large image area can be easily obtained, 
scattering throughout the image would hinder multivariate analysis without either correcting the spectra using existing algorithms, or using clustering to mask out those regions with the dispersion artefact from further analysis, which may exclude relevant parts of the tissue. Indeed, cancerous tissue with enlarged cell nuclei may be excluded should such a method be used. By contrast, Figure 4 shows the same region of tissue with a markedly reduced number of clusters containing the scattering artefact. The degree of scattering (i.e. the dispersion artefact near the band corresponding to Amide I) is also reduced within cluster 2 compared with the same cluster from Figure 3, which should therefore be more straightforward to correct using iterative algorithms. Importantly, this demonstrates that it is possible to combine lens-added approach with mapping, thus obtaining images with enhanced spatial resolution and reduced scattering while still imaging large areas of the sample.

Lastly, we performed hierarchical clustering analysis (HCA) to identify a region of high-grade dysplasia from the surrounding tissue. An adjacent section was stained to highlight the region of high-grade dysplasia as shown in Figure 5. Spectral bands within the $1300 \mathrm{~cm}^{-1}$ to $900 \mathrm{~cm}^{-1}$ region contain indicators for the presence of dysplasia and can therefore be used for classification. ${ }^{38,} 39$ Second derivative spectra (with 7 point smoothing) were obtained from the region between 1300 $\mathrm{cm}^{-1}$ to $1000 \mathrm{~cm}^{-1}$ and were vector normalised. To increase effective signal to noise, the spectral data cube was interpolated in the spatial domain by a reduction of $4 x$ and subsequently, HCA was performed using Pearson's function and Ward's algorithm. The results of HCA applied to the tissue sample measured without the lens are shown in Figure 6 . The second derivative spectrum shows a decrease in the trough at $1077 \mathrm{~cm}^{-1}$ as an indicator for high-grade dysplasia as shown in cluster 3 . For our configuration with the lens, there is approximately $9.4 \mathrm{~mm}$ of calcium fluoride. As such, it was not possible to obtain clustering results for the tissue imaged with the lens due to the decreased signal to noise ratio below $1200 \mathrm{~cm}^{-1}$.

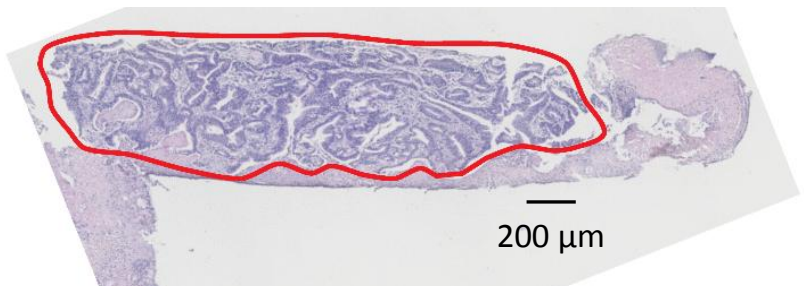

Figure $5-H+E$ staining of adjacent section of tissue sample with region marked as high-grade dysplasia. 


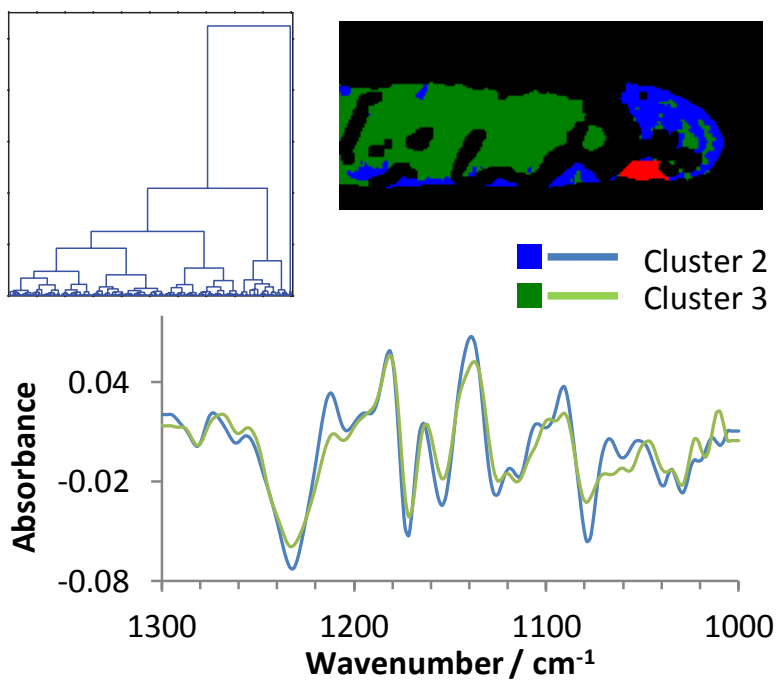

Figure 6 - HCA clustering of the tissue imaged without the lens, correctly indicating the high-grade dysplasia within the tissue. Average spectra from clusters 2 and 3 are plotted showing a decrease in the trough at $1077 \mathrm{~cm}^{-1}$ as one indicator of high-grade dysplasia. The average spectrum from the first cluster (red) is not plotted as it belongs to a corrupted data block, as also indicated the rightmost branch of the dendrogram.

\section{Assessment of spatial resolution for high-magnification FTIR imaging in transmission and FTIR imaging with the use of added lens using USAF resolution target}

The USAF target was used to assess the spatial resolution and field of view change with the use of lenses and high magnification. Figure 7 shows these results, with particular interest paid to the use of $\mathrm{HM}$ with and without the lens. It should be noted that without high magnification and without the lens, the projected pixel size of the Agilent Cary 620 is $5.5 \mu \mathrm{m}$, larger than that reported from the Bruker IRScope II, with a projected pixel size of $2.67 \mu \mathrm{m}$ under the same conditions. This trade-off in spatial resolution produces a larger field of view and is clear when comparing similar results from our previous work. ${ }^{25}$ Extracted transmission profiles from elements 4, 5 and 6 from group 7 in HM mode show that none of the elements are fully resolved, which is expected based on previous work $^{26}$ and the wavelength of infrared used $(3.2 \mu \mathrm{m})$ even with a projected pixel size of $1.1 \mu \mathrm{m}$. However, contrary to expectations, the contrast between the lines for each element imaged with the lens decreases compared to without the lens in HM mode. This may be due to optical phenomena within the system such as reflections at the interfaces between the lens, window and USAF target. The noise is also increased due to greater disparity between the condenser and objective lens numerical apertures resulting in a loss of throughput. These results show that for this particular configuration, using the lens does not increase the spatial resolution. 


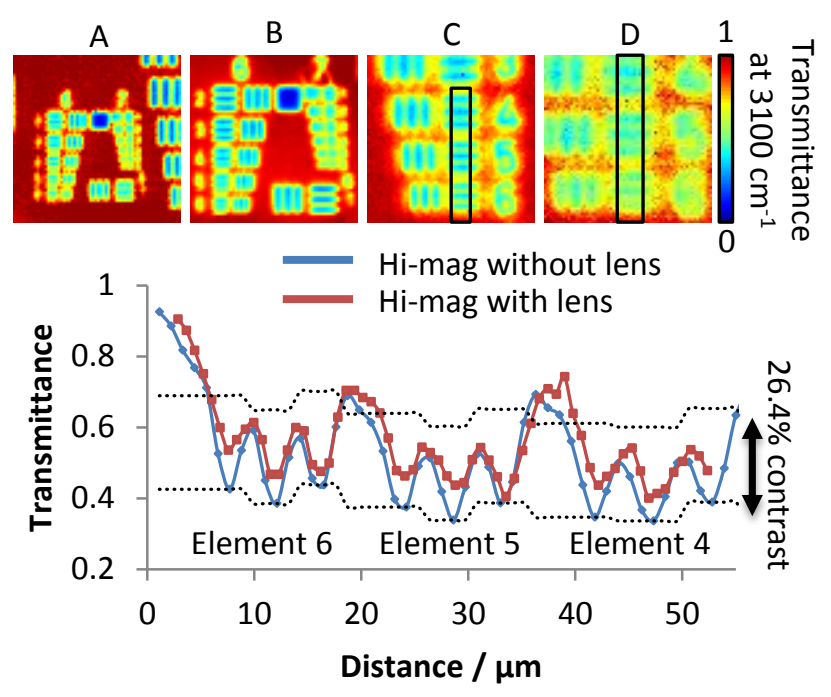

Figure 7-Transmission FTIR images at $3100 \mathrm{~cm}^{-1}$ of the USAF target without HM, without the lens (A), without HM, with the lens (B), with $H M$, without the lens $(C)$ and with $H M$, with the lens $(D)$. The HM measurements were focused on elements 4 , 5 and 6 of group 7. Regions of the HM images marked in black were extracted and averaged in the horizontal direction to produce transmittance profiles for the HM with and without the lens. Lines denoting the minimum contrast (26.4\%) considered as fully resolved are plotted from the troughs of the transmittance profiles.

\section{Assessment of spatial resolution for high-magnification FTIR imaging in transmission with the use of added lens with polymer interface sample and micro-ATR FTIR imaging}

It is important to assess the spatial resolution obtained using the lens and HM optics with a sample that contains two spectrally distinct parts with a sharp interface. A polymer laminate sample of PET and epoxy resin was used (with no interdiffusion between the PET and epoxy) ${ }^{40}$ and Figure 8 shows an absorbance image of the spectral band between $3251 \mathrm{~cm}^{-1}$ and $3448 \mathrm{~cm}^{-1}$ from using HM mode with top and bottom lenses, along with an integrated absorbance profile along the line perpendicular to the interface between two polymers to assess the spatial resolution, generated from averaging within the vertical direction of the image. A sigmoid function of the form shown in Eq. 2 was fitted to the integrated absorbance profile, and the coefficients $\left(c_{n}\right)$ were optimised to give an $\mathrm{R}^{2}$ value of 0.99 .

$$
f(x)=\frac{c_{1}}{c_{2}+e^{\left(-c_{3} x+c_{4}\right)}}+c_{5}
$$

Taking the limits of this function reveal the 'bulk' integrated absorbance of that spectral band within both halves of the sample. The apparent thickness of the interface was calculated as the distance between the points along the line perpendicular to interface at which the integrated absorbance values are between $10 \%$ and $90 \%$ of their maximum values. For this experiment gave the measured value of $12 \mu \mathrm{m}$. This is similar to our earlier work $(11 \mu \mathrm{m})^{25}$ obtained with a different imaging system and shows that the use lenses with HM mode does not increase spatial resolution - a similar result to that obtained with the USAF target. 


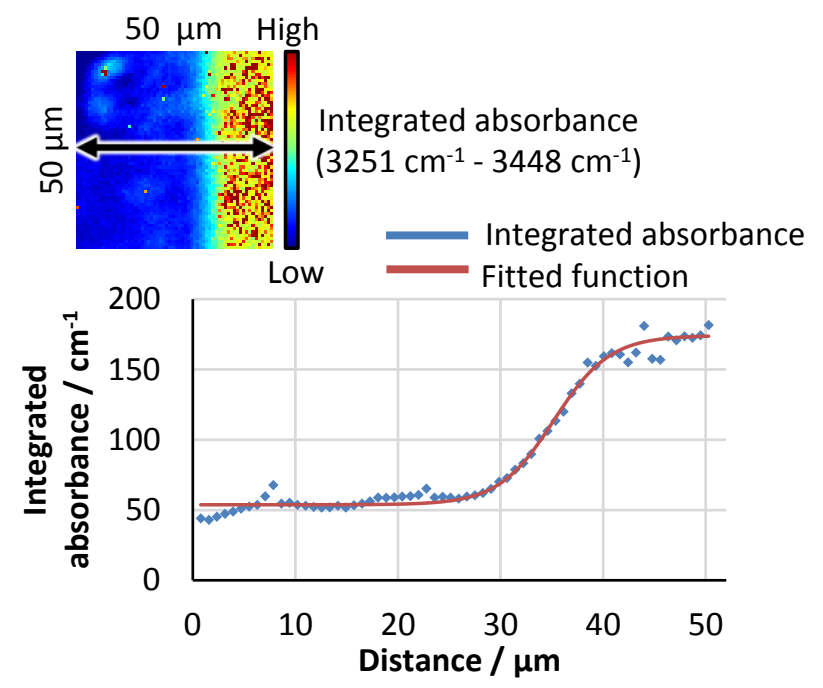

Figure 8 - FTIR image of polymer laminate obtained using transmission mode with HM and added lenses. The image is produced using the absorbance band between $3251 \mathrm{~cm}^{-1}$ and $3448 \mathrm{~cm}^{-1}$ and the integrated absorbance profile across the interface generated by averaging pixels in the vertical direction to produce a horizontal profile, as shown by the arrow. A sigmoid function is fitted and used to assess the spatial resolution.

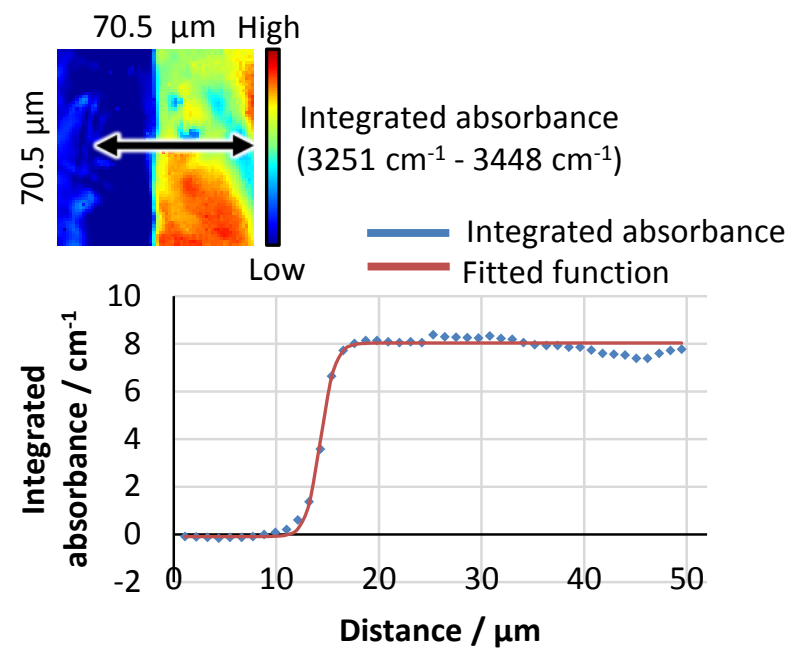

Figure 9 - FTIR image of polymer laminate obtained using micro-ATR mode with a germanium crystal. The image is produced using the absorbance band between $3251 \mathrm{~cm}^{-1}$ and $3448 \mathrm{~cm}^{-1}$ and the integrated absorbance profile across the interface generated by averaging pixels in the vertical direction to produce a horizontal profile, as shown by the arrow. A sigmoid function is fitted and used to assess the spatial resolution.

By contrast, micro-ATR measurements of the same polymer interface produce a sharper profile and using the same analysis gives an apparent interface thickness of $3 \mu \mathrm{m}$ (Figure 9). These results show that spatial resolution, when measured as a function of spectral contamination across a sharp interface, is often lower than that predicted by projected pixel size.

\section{Tissue measurements with high magnification and the lens}

To assess the effect of the lens with and without HM mode on the quality of spectra and degree of scattering from biological samples, single spectral images of the tissue were measured. Figure 10 shows the results of these measurements. It is clear from Figure $10 \mathrm{~A}$ and $\mathrm{C}$, scattering is reduced with the lens as the number of pixels classified as containing the scattering artefact is lower in C. However, in Figure $10 \mathrm{C}$, baseline undulations as a result of internal reflections between the 
windows are observed, though this type of interference is readily removable. ${ }^{41}$ With $\mathrm{HM}$ mode as shown in Figure $7 \mathrm{~B}$ and $\mathrm{D}$, the effect of the lens is less clear with respect to scattering, however the signal to noise is substantially worse with the lens, especially around $1000 \mathrm{~cm}^{-1}$, which is due to the mis-match between the condenser and objective numerical aperture, and the thickness of calcium fluoride attenuating frequencies below approximately $1200 \mathrm{~cm}^{-1}$. As concluded from the results above with the polymer interface sample, the use of HM mode and the lens does not yield any benefit, and decreases the signal to noise considerably. It was noted by Foreman et $\mathrm{al}^{37}$ and others, ${ }^{18,23}$ that the region between $1200 \mathrm{~cm}^{-1}$ and $900 \mathrm{~cm}^{-1}$ contains vital information on the disease state of the tissue, and that while light at these frequencies is attenuated, approaches using more powerful sources such as synchrotron light ${ }^{23}$ or quantum cascade lasers can be used to increase throughput in this region. ${ }^{42}$ Another solution to increase the light throughput of frequencies below $1200 \mathrm{~cm}^{-1}$ is to use alternative window materials instead of calcium fluoride. We attempted to use windows and lenses made from multispectral grade zinc sulphide (refractive index of 2.2) which would not only increase spatial resolution but still allow for alignment using visible light and increase transmission below $1200 \mathrm{~cm}^{-1}$. However, throughput was severely diminished, as predicted by the Fresnel equations which gives a total transmittance of approximately $54.5 \%$ for 4 surfaces, i.e. window and lens surfaces. Interference from internal reflections also severely hindered usability such that quality of spectra were significantly worse compared with using calcium fluoride. Transmittance through calcium fluoride for the same configuration is approximately $90 \%$. Optimising the thickness of the lenses and windows would also reduce attenuation of light at the mentioned frequencies. 
Amide II integrated absorbance $\left(1485 \mathrm{~cm}^{-1}\right.$ $-1570 \mathrm{~cm}^{-1}$ )

A

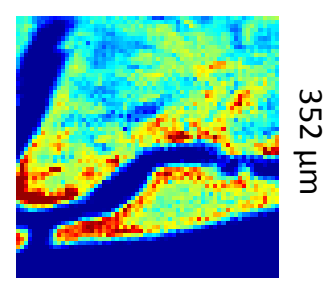

$352 \mu \mathrm{m}$
KMC clusters
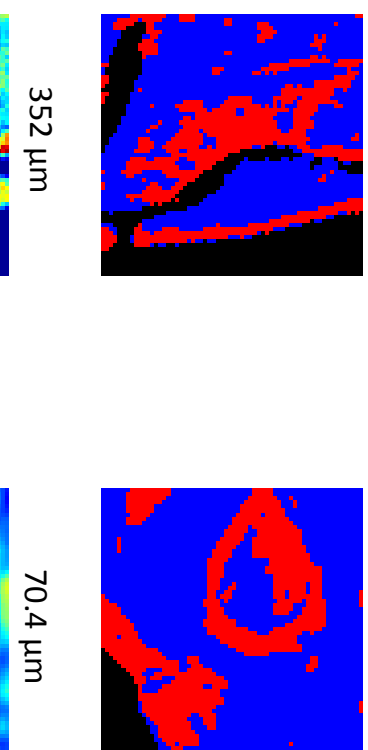

$70.4 \mu \mathrm{m}$

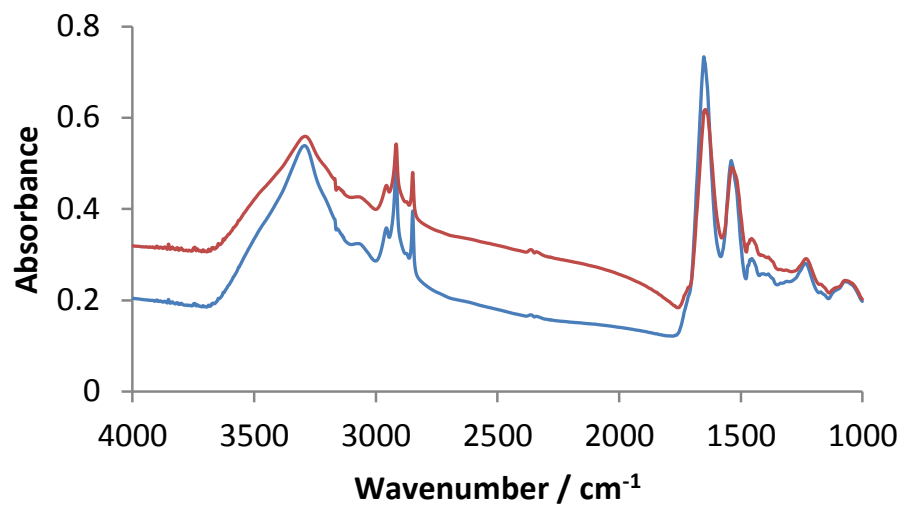

B

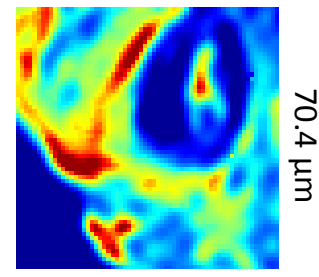

C

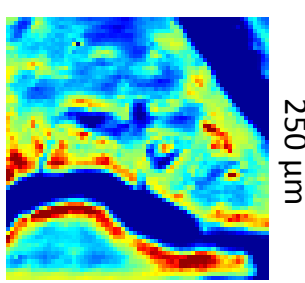

$250 \mu \mathrm{m}$

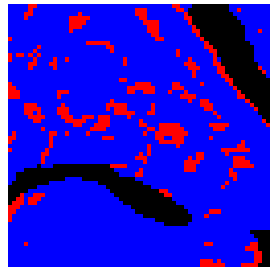

D

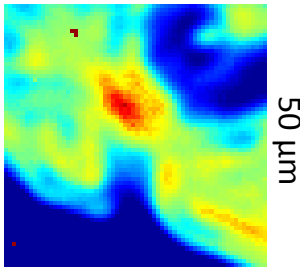

$50 \mu \mathrm{m}$

Integrated

Absorbance $/ \mathrm{cm}^{-1}$

High

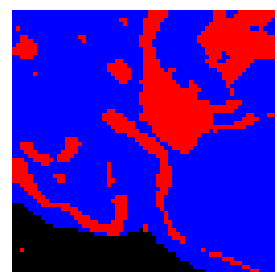

- Cluster 1

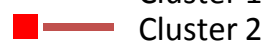

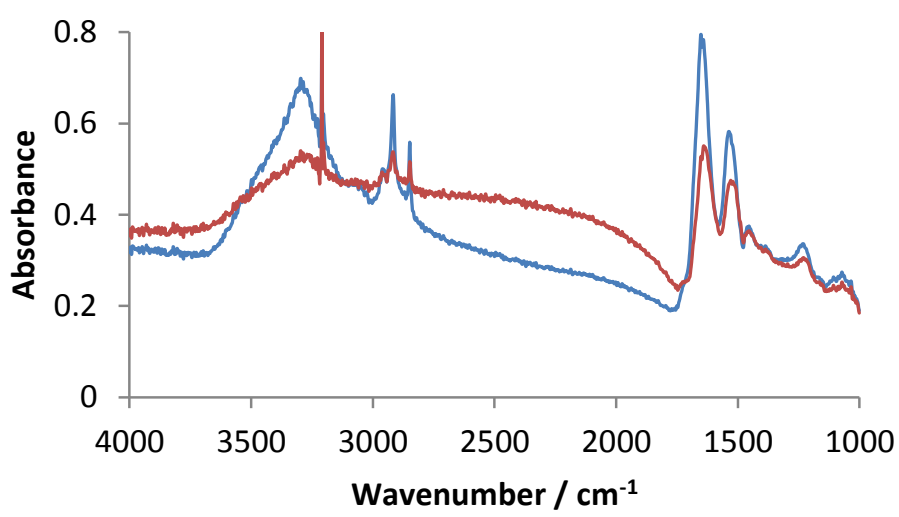
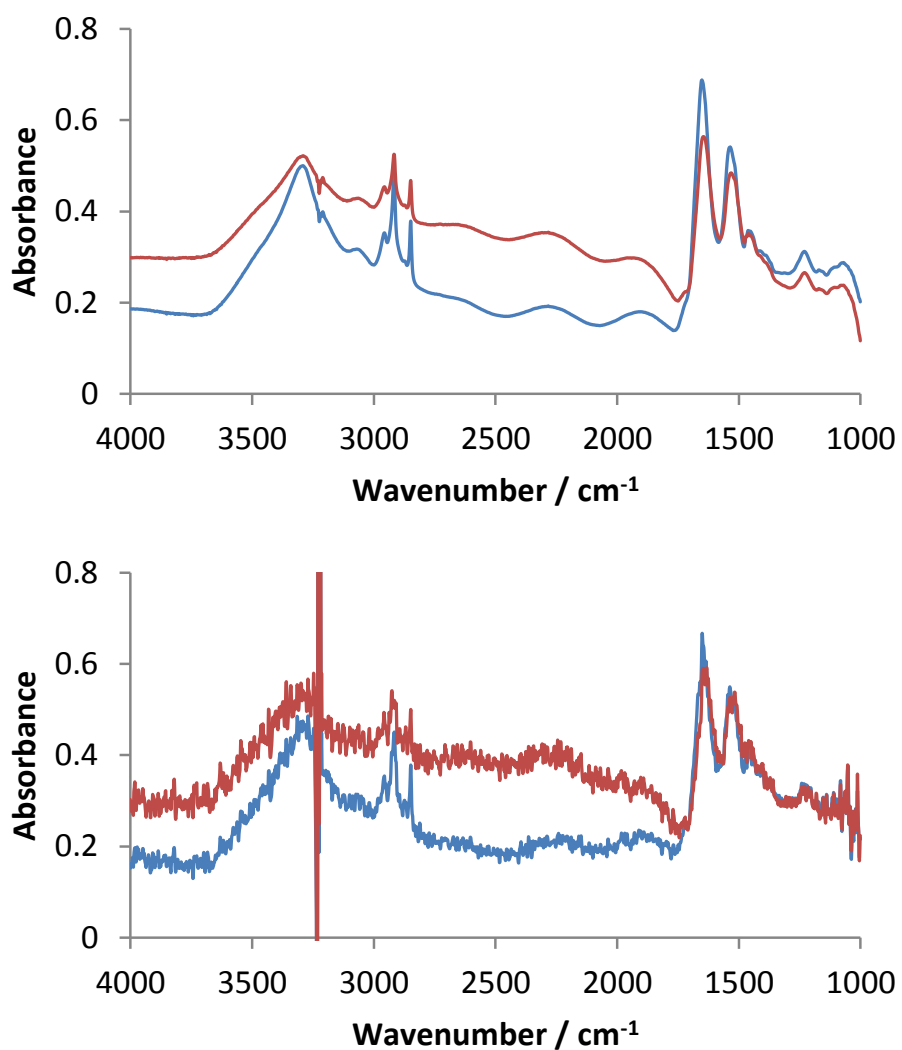

Figure 10 - Integrated absorbance images of tissue and clusters, and average spectra from each cluster imaged A) without $H M$, without lens, B) with $H M$, without lens, C) without $H M$, with lens and D) with $H M$, with lens. 


\section{Micro-ATR spectroscopic imaging of tissue}

Micro-ATR mode uses a crystal (commonly germanium) in contact with the sample through which infrared light undergoes total internal reflection. Diffraction effects are eliminated and the spatial resolution is enhanced due to the high refractive index of the ATR crystal. Using the same analytical approach, it can be seen that the dispersion artefact is completely removed and the second cluster identified is simply poorer quality spectra rather than spectra containing scattering (Figure 11). Within the fingerprint region, the depth of penetration of the evanescent wave is approximately 0.5 $\mu \mathrm{m}^{40}$ and is independent of sample thickness, allowing a variety of tissue samples to be studied without the need for microtoming. For biopsies, ATR mode does not require extensive preparation such as microtoming and deparaffinisation, which could affect the chemical properties of the sample.
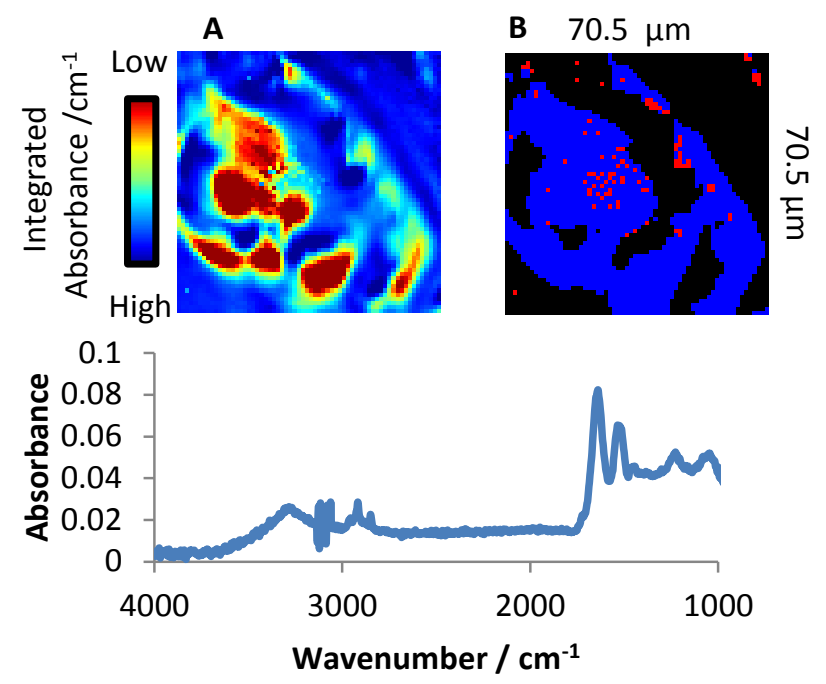

Figure 11 - Micro-ATR image of esophageal tissue (A) and KMC clusters (B). The graph shows the average spectrum from cluster 1 (blue regions), indicating the complete removal of scattering.

\section{Imaging of live cells}

Imaging live HEK 293A cells within microfluidic devices benefits greatly from the use of the lens as shown in Figure 12 and our previous work ${ }^{43}$. Spatially smoothing the spectra using a 3 pixel Gaussian filter and producing integrated absorbance images from the Amide-II band shows an increase in sharpness using the lens, making comparison with the visible images relatively straightforward. Cell morphology is easily assessed from spectroscopic images which is an indication of the state of health of the cells. For live biological systems and other microfluidics, the use of two windows is often unavoidable and the use of lenses to correct refraction is a practical way to improve significantly the spectral quality. 

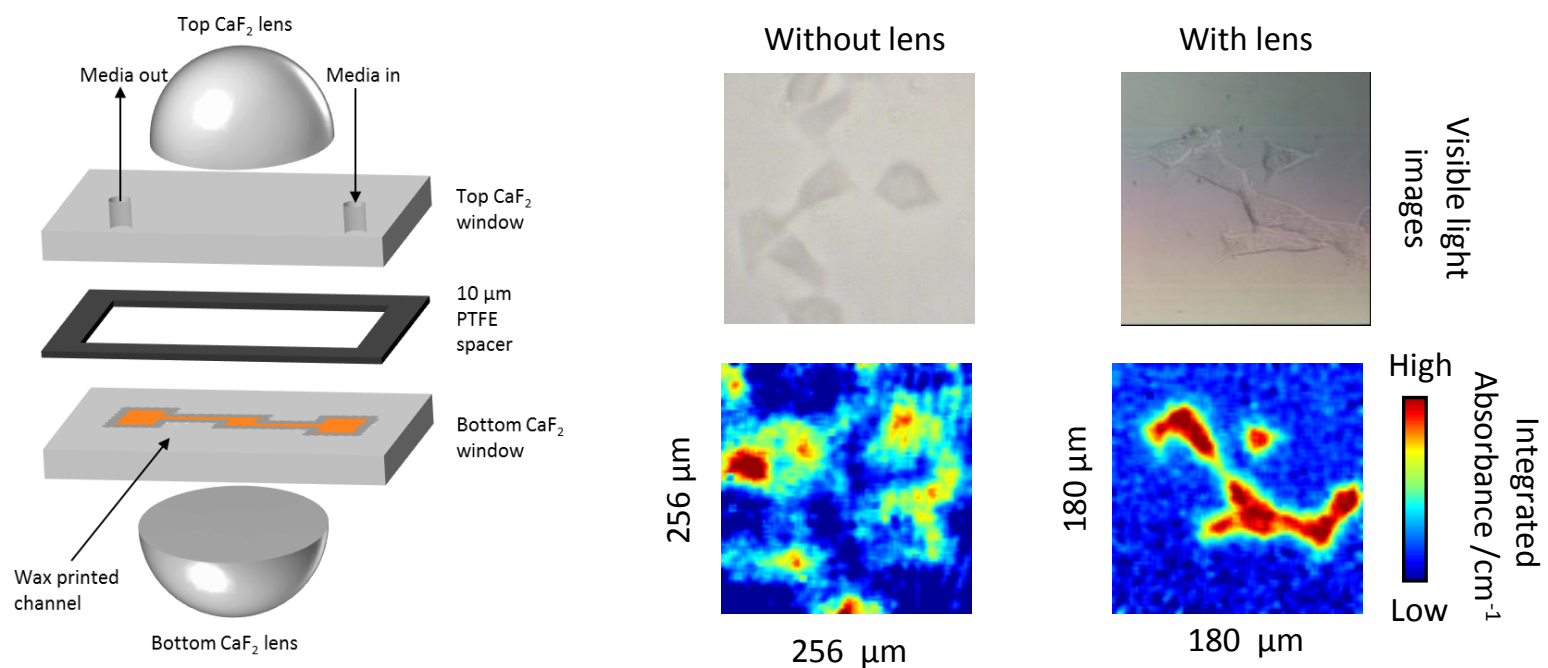

Figure 12 - Microfluidic device with live cells imaged with and without the lens through $4 \mathrm{~mm}$ thick calcium fluoride windows. The spectroscopic images are generated using the Amide II band between $1485 \mathrm{~cm}^{-1}$ and $1570 \mathrm{~cm}^{-1}$, with the benefit of the lens apparent compared to without.

\section{Conclusions}

Combining mapping and spectroscopic imaging of tissue mounted on a calcium fluoride window, with a calcium fluoride lens has been demonstrated. Using this method, it is possible to image large sections of tissue, correcting for chromatic aberration and removing scattering, as shown using kmeans clustering classification. As each tile is scanned separately and assembled post-scan, the method is reliable, allowing for maps of any size to be acquired before stitching and computational analysis. The reduction of scattering would assist in further computational processing of spectra to remove the remaining artefacts. HCA was able to correctly classify high-grade dysplasia from normal tissue for the map obtained without the lens but was unable to do so for the map obtained with the lens due to the decreased throughput of infrared light at frequencies below $1200 \mathrm{~cm}^{-1}$.

The Agilent Cary 620 microscope contains high-magnification (HM) optics, giving a projected pixel size of $1.1 \mu \mathrm{m}$ when coupled with a $15 x$ objective. In non-high-mag mode, the projected pixel size is larger $(5.5 \mu \mathrm{m})$ due to the use of expanding optics to give a larger field of view at the expense of spatial resolution. We show using the USAF target the change in field of view and also the performance of the lens coupled with the HM optics. It was shown that in cases with and without the lens, the last elements of group 7 are partially resolved. However, the contrast is slightly reduced using the lens compared to without. This may be due to internal reflections from the increased number of surfaces through which the infrared light passes, and small imperfections in the optical alignment of the lens. With a projected pixel size of $785 \mathrm{~nm}$, the noise is also increased, thus the full potential of using the lens with HM optics could only be realised using more powerful sources such as a synchrotron or quantum cascade laser.

As the USAF target represents a highly idealised system, a sample containing a sharp interface between two polymers was imaged in transmission using both a top and a bottom lens. This method using two lenses corrected chromatic aberration for the condenser and the objective, maximising the potential spatial resolution that could be obtained. Using the integrated absorbance of a spectral band present in one polymer but not the other, a profile was obtained across the image. 
The change in integrated absorbance from one polymer to the other indicates the useful spatial resolution of the system, as the objective of spectroscopic imaging is to spatially resolve spectrally dissimilar regions i.e. where spectral features of one are absent in the other. The width of the interface was shown to be $12 \mu \mathrm{m}$, similar to that observed in previous work and nevertheless, higher than the theoretical maximum resolution. The oversampling capability of the HM optics thus provides no discernible increase in spatial resolution when used with the lens. Importantly, MicroATR spectroscopic imaging of the same interface show higher resolution is obtained (3.3 $\mu \mathrm{m})$ in spite of larger projected pixel size, demonstrating the importance of measuring real samples to assess spatial resolution rather than assuming the projected pixel size is the spatial resolution.

Spectral images of the tissue section with and without the lens, and with and without HM optics were analysed using k-means clustering. Without HM optics, the reduction in scattering was evident from the averaged spectra. However, with HM optics, scattering is not reduced significantly, and signal to noise is significantly worse, precluding analysis for diagnostic purposes and, should mapping be performed, increasing the time to map an area significantly. The highest performance of the lens can therefore be obtained using a $15 x$ objective alone, reducing scattering and achieving sufficient signal to noise. To address the loss of throughput at the lower frequencies, which are important for clinical diagnostics, thinner windows and lenses could be used ${ }^{14}$. For comparison with transmission images, spectra were obtained from the same sample using a micro-ATR germanium crystal. Spectra from k-means clustering showed the absence of scattering and demonstrated the feasibility of using ATR mode to obtain high-quality spectra and can be used for similar studies without the need for microtoming.

This approach could also be applied to other devices such as microfluidic systems, which often operate at quasi-steady state. With these systems, transmission through spectroscopic windows cannot be avoided, hence the lens is particularly useful to remove chromatic aberration. We present a microfluidic device containing live HEK cells, imaged with and without the lens. With the lens, the correlation with the visible image is straightforward and the cell boundary is clear, aiding in the assessment of their morphology. The use of lenses and the mapping approach would in future allow the entire device to be studied at high resolution.

1. R. Salzer and H. W. Siesler, eds., Infrared and Raman Spectroscopic Imaging, 2nd Edition, Wiley-VCH Verlag GmbH \& Co. KGaA, Weinheim, 2014.

2. S. Šašić and Y. Ozaki, eds., Raman, Infrared, and near-infrared chemical imaging, John Wiley \& Sons, Inc., Hoboken, New Jersey, 2010.

3. S. G. Kazarian and K. L. A. Chan, Applied Spectroscopy, 2010, 64, 135A-152A.

4. G. Steiner and E. Koch, Anal Bioanal Chem, 2009, 394, 671-678.

5. O. S. Fleming, K. L. A. Chan and S. G. Kazarian, Polymer, 2006, 47, 4649-4658.

6. C. Vogel, E. Wessel and H. W. Siesler, Biomacromolecules, 2008, 9, 523-527.

7. A. Gupper, K. L. A. Chan and S. G. Kazarian, Macromolecules, 2004, 37, 6498-6503.

8. J. L. Koenig, Microspectroscopic Imaging of Polymers, American Chemical Society, 1998.

9. A. A. Gabrienko, C. H. Lai and S. G. Kazarian, Energy \& Fuels, 2014, 28, 964-971.

10. F. H. Tay and S. G. Kazarian, Energy \& Fuels, 2009, 23, 4059-4067.

11. K. L. A. Chan and S. G. Kazarian, Analytical Chemistry, 2012, 84, 4052-4056.

12. K. L. A. Chan, X. Niu, A. J. deMello and S. G. Kazarian, Analytical Chemistry, 2011, 83, 36063609.

13. E. Carter, K. Tam, R. Armstrong and P. Lay, Biophys Rev, 2009, 1, 95-103. 
14. K. L. Andrew Chan and S. G. Kazarian, Chemical Society Reviews, 2015, DOI: 10.1039/C5CS00515A.

15. L. M. Miller, M. W. Bourassa and R. J. Smith, Biochimica et Biophysica Acta (BBA) Biomembranes, 2013, 1828, 2339-2346.

16. S. G. Kazarian and K. L. A. Chan, Analyst, 2013, 138, 1940-1951.

17. P. Gelfand, R. J. Smith, E. Stavitski, D. R. Borchelt and L. M. Miller, Analytical Chemistry, 2015, 87, 6025-6031.

18. C. Petibois and G. Deleris, Trends in Biotechnology, 2006, 24, 455-462.

19. R. Bhargava, Applied spectroscopy, 2012, 66, 1091-1120.

20. B. Bird, M. Miljkovic, S. Remiszewski, A. Akalin, M. Kon and M. Diem, Lab Invest, 2012, 92, 1358-1373.

21. C. Hughes and M. J. Baker, Analyst, 2015, DOI: 10.1039/C5AN01858G.

22. D. C. Fernandez, R. Bhargava, S. M. Hewitt and I. W. Levin, Nature Biotechnology, 2005, 23, 469-474.

23. L. M. Miller and P. Dumas, Biochimica et Biophysica Acta (BBA) - Biomembranes, 2006, 1758, 846-857.

24. J. A. Dougan, K. L. A. Chan and S. G. Kazarian, in Infrared and Raman Spectroscopic Imaging, Wiley-VCH Verlag GmbH \& Co. KGaA, 2014, DOI: 10.1002/9783527678136.ch9, pp. 397-444.

25. K. L. A. Chan and S. G. Kazarian, Analytical Chemistry, 2013, 85, 1029-1036.

26. C. R. Findlay, R. Wiens, M. Rak, J. Sedlmair, C. J. Hirschmugl, J. Morrison, C. J. Mundy, M. Kansiz and K. M. Gough, Analyst, 2015, 140, 2493-2503.

27. S. Inoué, in Handbook Of Biological Confocal Microscopy, ed. J. B. Pawley, Springer US, 2006, DOI: 10.1007/978-0-387-45524-2 1, ch. 1, pp. 1-19.

28. D. Mayerich, T. van Dijk, M. J. Walsh, M. V. Schulmerich, P. S. Carney and R. Bhargava, Analyst, 2014, 139, 4031-4036.

29. A. Kohler, J. Sulé-Suso, G. D. Sockalingum, M. Tobin, F. Bahrami, Y. Yang, J. Pijanka, P. Dumas, M. Cotte, D. G. van Pittius, G. Parkes and H. Martens, Applied Spectroscopy, 2008, 62, 259266.

30. P. Bassan, A. Kohler, H. Martens, J. Lee, H. J. Byrne, P. Dumas, E. Gazi, M. Brown, N. Clarke and P. Gardner, Analyst, 2010, 135, 268-277.

31. R. K. Reddy, M. J. Walsh, M. V. Schulmerich, P. S. Carney and R. Bhargava, Applied Spectroscopy, 2013, 67, 93-105.

32. V. Zohdi, D. R. Whelan, B. R. Wood, J. T. Pearson, K. R. Bambery and M. J. Black, PLoS ONE, 2015, 10, e0116491.

33. B. Mohlenhoff, M. Romeo, M. Diem and B. R. Wood, Biophysical Journal, 2005, 88, 36353640 .

34. P. Bassan, A. Sachdeva, A. Kohler, C. Hughes, A. Henderson, J. Boyle, J. H. Shanks, M. Brown, N. W. Clarke and P. Gardner, Analyst, 2012, 137, 1370-1377.

35. P. Bassan, A. Sachdeva, J. H. Shanks, M. D. Brown, N. W. Clarke and P. Gardner, Analyst, 2013, 138, 7066-7069.

36. A. Sroka-Bartnicka, J. Kimber, L. Borkowski, M. Pawlowska, I. Polkowska, G. Kalisz, A. Belcarz, K. Jozwiak, G. Ginalska and S. Kazarian, Anal Bioanal Chem, 2015, 407, 7775-7785.

37. L. Foreman, J. A. Kimber, K. V. Oliver, J. M. Brown, S. M. Janes, T. Fearn, S. G. Kazarian and P. Rich, 2015.

38. T. D. Wang, G. Triadafilopoulos, J. M. Crawford, L. R. Dixon, T. Bhandari, P. Sahbaie, S. Friedland, R. Soetikno and C. H. Contag, Proceedings of the National Academy of Sciences of the United States of America, 2007, 104, 15864-15869.

39. L. Quaroni and A. G. Casson, Analyst, 2009, 134, 1240-1246.

40. K. L. A. Chan and S. G. Kazarian, Applied Spectroscopy, 2003, 57, 381-389.

41. A. M. A. Pistorius and W. J. DeGrip, Vibrational Spectroscopy, 2004, 36, 89-95.

42. P. Bassan, M. J. Weida, J. Rowlette and P. Gardner, Analyst, 2014, 139, 3856-3859. 
43. K. L. A. Chan and S. G. Kazarian, Analyst, 2013, 138, 4040-4047. 